\title{
An evaluation of 2.0 McFarland Etest method for detection of heterogeneous vancomycin-intermediate Staphylococcus aureus
}

\author{
Montri Pongkumpai ${ }^{\mathrm{a}}$, Suwanna Trakulsomboon ${ }^{\mathrm{b}}$, Chusana Suankratay ${ }^{\mathrm{a}}$ \\ ${ }^{a}$ Division of Infectious Diseases, Department of Medicine, Faculty of Medicine, Chulalongkorn \\ University, Bangkok 10330; 'Division of Infectious Disease and Tropical Medicine, Department of \\ Medicine, Faculty of Medicine, Siriraj Hospital, Mahidol University, Bangkok 10700, Thailand
}

\begin{abstract}
Background: Staphylococcus aureus with reduced susceptibility to vancomycin or heterogeneous vancomycinintermediate $S$. aureus (hVISA) have become increasingly reported from various parts of the world. hVISA cannot be detected by routine test for minimal inhibitory concentration (MIC) for vancomycin. The gold standard method for detection, population analysis profiles (PAP) method, is complicated, time-consuming, expensive, and needs well-trained microbiologists.

Objective: Evaluate of 2.0 McFarland Etest method, in comparison with the PAP method, for detection of hVISA in clinical specimens.

Methods: All methicillin-resistant S. aureus strains from clinical specimens isolated from consecutive patients attended at King Chulalongkorn Memorial Hospital and Siriraj Hospital, Bangkok between 2006 and 2007 were studied. 1 hundred nineteen specimens were obtained. The PAP method detected six hVISA strains 5 from blood and from cultures) from four patients at King Chulalongkorn Memorial Hospital, accounting for a prevalence of $6.35 \%$. The MIC determined by agar dilution method was in the range of $2-3 \mu \mathrm{g} / \mathrm{mL}$.

Results: 2.0 McFarland Etest method detected no false positive and five false negatives (42\%), and gave a sensitivity and a specificity of $16.7 \%$ and $100 \%$, respectively. The one-point population analysis screening method detected two false positives and 1 false negative, and gave a sensitivity of $83.3 \%$ and a specificity and $98.2 \%$.

Conclusion: The 2.0 McFarland Etest method had a very good specificity but a poor sensitivity for detecting hVISA. It may be used as an alternative method to confirm detection of hVISA.
\end{abstract}

Keywords: Etest, heterogeneous vancomycin-intermediate $S$. aureus, population analysis profiles, Staphylococcus aureus, susceptibility

Methicillin-resistant Staphylococcus aureus (MRSA) infections are a major health problem worldwide. The increasing use of glycopeptides makes the organism more virulent and resistant [1]. S. aureus with reduced susceptibility to glycopeptides was first reported from Japan in 1996 in a patient with MRSA pneumonia [2, 3]. Since then, similar strains have been isolated in the United States [4],

Correspondence to: Chusana Suankratay MD, PhD, Division of Infectious Diseases, Department of Medicine, Faculty of Medicine, Chulalongkorn University, Bangkok 10330, Thailand. Email: chusana.s@chula.ac.th
Europe [5], Asia [6], and Thailand [7]. These isolates display vancomycin minimal inhibitory concentration (MIC) below the breakpoint, but contain some bacterial subpopulations $\left(>10^{-6}\right)$ growing in the presence of $\geq 4 \mu \mathrm{g} / \mathrm{mL}$ of vancomycin. These isolates are termed heterogeneous vancomycin-intermediate S. aureus (hVISA). With the revised Clinical and Laboratory Standards Institute (CLSI) breakpoints for vancomycin [8], most hVISA strains with the MICs in the range of 3-6 $\mu \mathrm{g} / \mathrm{mL}$ may be reclassified as homogeneous VISA or VISA, making the differentiation between these strains less important than the accurate determination of hVISA strains with 
the MICs between 1-2 $\mu \mathrm{g} / \mathrm{mL}$, which would be identified as vancomycin-susceptible $S$. aureus (VSSA).

Recently, a SENTRY study showed that four of 253 MRSA strains were hVISA with the MICs between $1-2 \mu \mathrm{g} / \mathrm{mL}$ [9]. A recent study of 152 MRSA reported that $90 \%$ had vancomycin MIC of $\geq 2 \mu \mathrm{g} /$ $\mathrm{mL}$ as determined by Etest strips, while $9.8 \%$ exhibited vancomycin of $3 \mu \mathrm{g} / \mathrm{mL}$ [10]. In addition, hVISA strains may represent a gateway to high-level vancomycin resistance as VISA and vancomycinresistant $S$. aureus (VRSA) strains [11, 12]. All these evidences emphasize an urgent need for a suitable and reliable method to detect hVISA with vancomycin MICs of $1-2 \mu \mathrm{g} / \mathrm{mL}$, especially isolated from patients who fail to respond to glycopeptide treatment.

The population analysis profiles (PAP) method is generally used for detecting hVISA, but it is complicated, time-consuming, expensive, and needs well-trained microbiologists [2]. Using a laboratory method with comparable sensitivity and specificity but more convenience, simpler, and cheaper would be an alternative to the PAP. A modification of the Etest system with a heavy inoculum $[200 \mu \mathrm{L}$ of bacterial suspensions with the density equivalent to a 2.0 McFarland turbidity $\left(6 \times 10^{8}\right.$ colony-forming units $\left.\left./ \mathrm{mL}\right)\right]$ may improve the determination of hVISA strains. In this study, we aimed to evaluate the Etest at 2.0 McFarland inocula, in comparison with the PAP method, for detection of $h$ VISA in clinical specimens.

\section{Materials and methods}

Reference bacterial strains used were Mu50 (VISA) and Mu3 (hVISA) [2]. One hundred three clinical isolates of MRSA were consecutively obtained from patients hospitalized at King Chulalongkorn Memorial Hospital between January 2006 and March 2007. In addition, 16 additional clinical isolates of MRSA were collected from patients at Siriraj Hospital between May and November 2006. All MRSA isolates were tested for antimicrobial susceptibility by the diskdiffusion method according to the CLSI guidelines [8], and stored in skim mild glycerol at $-70^{\circ} \mathrm{C}$. All methods described in this study, apart from PAP method, were carried out in duplicate. Vancomycin standard powder was purchased from Sigma (Steinheim, Germany).

\section{One-point population analysis}

The one-point population analysis method was carried out as previously described by Hiramatsu et al. [2]. Specific bacterial inocula were plated onto brain heart infusion (BHI) agar containing $4 \mu \mathrm{g} / \mathrm{mL}$ of vancomycin. Growth of bacteria at 24 hours was considered a VISA strain. Growth occurring between 24 and 48 hours was considered a hVISA strain.

\section{PAP}

The PAP method was performed as previously described by Hiramatsu et al. [2]. $100 \mu \mathrm{L}$ of a bacterial suspension [made by adjusting an overnight culture in BHI broth to optical density $578 \mathrm{~nm}$ of 0.3 (about $10^{8}$ colony-forming units/mL) with fresh BHI broth]. Tenfold dilutions of this suspension were plated onto BHI agar plates containing various concentrations of vancomycin. After incubation at $37^{\circ} \mathrm{C}$ for 48 hours, the number of colonies grown on each plate was counted. The number of resistant cells contained in $100 \mu \mathrm{L}$ of the starting cell suspension was calculated and plotted on a semilogarithmic graph.

\section{Etest}

Etest MICs were determined using two inoculum densities of 0.5 and 2.0 McFarland [13]. Strains were grown overnight on blood agar plates. A randomly selected single colony was inoculated into fresh Mueller-Hinton broth, and grown overnight. The turbidity of the broth was adjusted to 0.5 and 2.0 McFarland standards using fresh broth. Then, $200 \mu \mathrm{L}$ of this suspension was pipetted onto a $90-\mathrm{mm}$ BHI agar (BBL, Becton Dickinson, Cockeysville, USA) plate and streaked out evenly with a swab. After being dried for approximately 10 minutes, Etest strips (AB BIO Disk) for vancomycin $(0.06-256 \mu \mathrm{g} / \mathrm{mL})$ were applied. All plates were incubated at $35^{\circ} \mathrm{C}$ for 24 hours.

\section{Agar dilution}

The method was carried out as recommended by the CLSI guidelines [14]. Colonies were taken from overnight blood agar plates, and sterile saline was inoculated to make a $0.5 \mathrm{McF}$ arland suspension inoculum. Mueller-Hinton agar(Oxoid) was inoculated with the suspension inoculum. The plates were incubated at $35^{\circ} \mathrm{C}$ and read after 24-hour incubation.

\section{Analysis}

The performance of 2.0 McFarland Etest and onepoint population analysis methods in detecting hVISA was determined, in comparison with the PAP method. Each method was evaluated for sensitivity and specificity in discriminating hVISA form MRSA 
strains. The sensitivity was calculated based on the number of hVISA that were correctly identified, while the specificity was calculated based on the number of correct negative results. The positive predictive value (PPV) refers to the probability that a positive result is correct, while the negative predictive value (NPV) refers to the probability that a negative result is correct.

\section{Results}

One hundred nineteen clinical specimens were obtained during the study period. The PAP method detected 6 hVISA strains (five and one from blood and pus cultures, respectively) from four patients at King Chulalongkorn Memorial Hospital, accounting for a prevalence of $6.35 \%$ among MRSA isolates.

The performance of 2.0 McFarland Etest and one-point population analysis methods is shown in
Table 1. 2.0 McFarland Etest method detected no false positive $(0 \%)$ and five false negatives $(42 \%)$, hence showing a sensitivity of $16.7 \%$, a specificity of $100 \%$, a PPV of $100 \%$, and an NPV of $95.8 \%$. The one-point population analysis screening method detected two false positives and one false negative, hence giving a sensitivity of $83.3 \%$, a specificity of $98.2 \%$, a PPV of $71.4 \%$, and an NPV of $99.1 \%$.

The vancomycin MIC of five hVISA strains determined by agar dilution method was $2 \mu \mathrm{g} / \mathrm{mL}$, and one strain isolated from the blood had the MIC of $3 \mu \mathrm{g} / \mathrm{mL}$ (Table 2). In addition, all these six hVISA strains had the MICs determined by 0.5 McFarland Etest method between 1.5 and $3 \mu \mathrm{g} / \mathrm{mL}$, which were in the susceptible range based on the CLSI breakpoint criteria for vancomycin. The $2.0 \mathrm{McF}$ arland Etest method detected two of these six strains with the MIC of 3 and $6 \mu \mathrm{g} / \mathrm{mL}$.

Table 1. Sensitivity and specificity of one-point population analysis, 2.0 McFarland Etest method, in comparison with the population analysis profile method, in detecting heterogeneous vancomycin-intermediate Staphylococcus aureus.

\begin{tabular}{lcccccc}
\hline Method & $\begin{array}{c}\text { False } \\
\text { positive } \\
\text { (number) }\end{array}$ & $\begin{array}{c}\text { False } \\
\text { negative } \\
\text { (number) }\end{array}$ & Sensitivity & Specificity & PPV & NPV \\
(percent) & (percent) & (percent) & (percent) \\
\hline 2.0 McFarland Etest & 0 & 5 & 16.7 & 100 & 100 & 95.8 \\
$\begin{array}{l}\text { One-point population } \\
\text { analysis }\end{array}$ & 2 & 1 & 83.3 & 98.2 & 71.4 & 99.1 \\
\hline
\end{tabular}

PPV: positive predictive value, NVP: negative predictive value

Table 2. One-point population analysis, population analysis profile (PAP) and minimal inhibitory concentration (MIC) determined by agar dilution and 2.0 McFarland and Etest methods of all 6 heterogeneous vancomycin-intermediate Staphylococcus aureus.

\begin{tabular}{cllcccc}
\hline Isolate & Specimen & $\begin{array}{c}\text { One-point } \\
\text { population } \\
\text { analysis }\end{array}$ & PAP & $\begin{array}{c}\text { MIC by } \\
\text { agar dilution } \\
\text { method } \\
(\boldsymbol{\mu g} / \mathbf{m L})\end{array}$ & $\begin{array}{c}\text { MIC by } \\
\text { 0.5 McFarland } \\
\text { Etest } \\
(\mu \mathrm{g} / \mathbf{m L})\end{array}$ & $\begin{array}{c}\text { MIC by } \\
\text { 2.0 McFarland } \\
\text { Etest } \\
(\mu \mathrm{g} / \mathbf{m L})\end{array}$ \\
\hline 1. & Blood & Positive & Positive & 2 & 2 & 3 \\
2. & Blood & Negative & Positive & 2 & 1.5 & 2 \\
3. & Blood & Positive & Positive & 2 & 2 & 2 \\
4. & Blood & Positive & Positive & 2 & 2 & 2 \\
5. & Pus & Positive & Positive & 2 & 2 & 6 \\
6. & Blood & Positive & Positive & 3 & 3 & 6 \\
\hline
\end{tabular}




\section{Discussion}

The detection of hVISA by routine susceptibility testing is unreliable, and this strain is most probably being underreported $[11,12]$. Standardized reference methods for susceptibility testing (agar dilution method recommended by the CLSI) also performed suboptimally in detecting hVISA, even with the recently revised MIC breakpoint criteria for VISA with the MIC of 4-8 $\mu \mathrm{g} / \mathrm{mL}$. Most of hVISA strains have the MICs between 1 and $2 \mu \mathrm{g} / \mathrm{mL}$, and hence would be identified as VSSA in clinical laboratory. Our results clearly confirmed this statement. All hVISA strains tested had MICs determined by agar dilution method in the susceptible range between 1.5 and $3 \mu \mathrm{g} / \mathrm{mL}$.

Even though the PAP method represents the gold standard for detecting hVISA strains, a use of this method in the clinical laboratory will be inconvenient, time-consuming, expensive, and needs well-trained microbiologists. A simpler and reliable clinical laboratory test is urgently needed. Different strategies of susceptibility testing have been used to detect hVISA strain including the use of different culture media, higher bacterial inocula, longer incubation periods before obtaining of susceptibility results, and lower vancomycin concentrations. Previous studies evaluated several methods for detection of both VISA and hVISA, in comparison with the PAP method [13, $15,16]$. To the best of our knowledge, there has been only one small study by Lulitanond and colleagues to evaluate the disk diffusion method with lowconcentration vancomycin disk for detecting hVISA strains [17]. In this prospective study with a larger sample size, we use two strategies including a high bacterial inoculum and the simple vancomycin Etest strips for detection of hVISA. Our results showed that the 2.0 McFarland Etest method had a $100 \%$ specificity and PPV, but a very low sensitivity, if the MIC breakpoints were between 4 and $8 \mu \mathrm{g} / \mathrm{mL}$. This is in contrast with the results by Walsh and colleagues, which showed that the sensitivity and specificity of the $2.0 \mathrm{McF}$ arland Etest method was $96 \%$ and $97 \%$, respectively. However, they included both VISA and hVISA strains in their study. It is our opinion that after the recent revision of the CLSI MIC breakpoints for VISA to $4-8 \mu \mathrm{g} / \mathrm{mL}$, most of hVISA strains in their study may be reclassified as VISA, and hence may be not difficult for differentiation from VSSA strains in a clinical laboratory. It seems that the $2.0 \mathrm{McFarland}$ Etest method has the disadvantage of lower sensitivity and produces higher false negative results in comparison with the one-point population analysis method. It should not be used as a screening test for detection hVISA. A convenient and reliable method for screening of hVISA is still needed for clinical laboratory.

In conclusion, this is the first prospective study to evaluate the $2.0 \mathrm{McF}$ arland Etest method for detection of hVISA without including homogeneous VISA. The 2.0 McFarland Etest method has a very good specificity but a poor sensitivity for detection of hVISA. Due to its high convenience and lower cost in comparison with the PAP method, it may be used as an alternative method to confirm the detection of hVISA.

\section{Acknowledgments}

We thank Mrs. Sumanee Nilgate of Department of Microbiology, Faculty of Medicine, Chulalongkorn University for her assistance with this study.

We have no conflict of interest to report.

\section{References}

1. Mayhall CG. Prevention and control of vancomycin resistance in Gram-positive coccal microorganism: fire prevention and fire fighting. Infect Control Hosp Epidemiol. 1996; 17:353-5.

2. Hiramatsu K, Aritaka N, Hanaki H, Kawasaki S, Hosoda Y, Hori S, et al. Dissemination in Japanese hospitals of strains of Staphylococcus aureus heterogeneously resistant to vancomycin. Lancet. 1997;350:1670-3.

3. Centers for Disease Control and Prevention. Reduced susceptibility of Staphylococcus aureus to vancomycin-Japan 1996. Morbl Mortal Wkly Rep. 1997. 46:624-6.

4. Rybak MJ, Chin JN, Lau K, Sader KH, Jones RN. Increasing prevalence of glycopeptide heteroresistant $S$. sureus from the Detroit metropolitan area over a 20-year period (1986-2006). Abstract $17^{\text {th }}$ Eur Congr Clin Microbiol Infect Dis (ECCMID), 2007; 032.

5. Ploy MC, Grelaud C, Martin CC, de Lumley L, Dennis F. First clinical isolate of vancomycin-intermediate Staphylococcus aureus in a French hospital. Lancet. 1998.351:1212.

6. Wong SS, Ho PL, Woo PC, Yuen KY. Bacteremia caused by staphylococci with inducible vancomycin heteroresistance. Clin Infect Dis. 1999; 29:760-7.

7. Trakulsomboon S. Danchaivijitr S, Rongrungruang Y, Dhiraputra C, Susaemgrat W, Ito T, et al. First report 
of methicillin-resistant Staphylococcus aureus with reduced susceptibility to vancomycin in Thailand. J Clin Microbiol. 1999; 39:591-5.

8. Clinical and Laboratory Standards Institute. Performance standards for antimicrobial susceptibility testing. Sixteen informational supplement. Approved standard M100-S16. Clinical and Laboratory Standards Institute, Wayne, USA. 2006.

9. Howe RA, Noel AR, Bowker KE. 2003. The prevalence of heterogeneously vancomycin intermediate Staphylococcus aureus (hVISA) in the USA. Abstract 43rd Interscience Conference on Antimicrobial Agents and Chemotherapy (ICAAC), 2003; abstr. C2-1828.

10. Delgado AJ, Riordan T, Lamichhane-Khadka R, Winnett DC, Jimenez J, Robinson K, et al. Heterovancomycin-intermediate methicillin-resistant Staphylococcus aureus isolate from a medical center in Las Cruces, New Mexico. J Clin Microbiol. 2007; 45: 1325-9.

11. Goldstein F. The potential clinical impact of low-level antibiotic resistance in Staphylococcus aureus. J Antimicrob Chemother. 2007; 59:1-4.

12. Appelbaum PC. Reduced glycopeptide susceptibility in methicillin-resistant Staphylococcus aureus (MRSA). Int J Antimicrob Agents. 2007; 30:398-408.

13. Walsh TR, Bolmström A, Qwärnström A, Ho P,
Wootton M, Howe RA, et al. Evaluation of current methods for detection of staphylococci with reduced susceptibility to glycopeptides. J Clin Microbiol. 2001; 39:2439-2444.

14. National Committee for Clinical Laboratory Standards. 2000. Methods for dilution antimicrobial susceptibility tests for bacteria that aerobically, 5th ed. Approved standard M7-A5. National Committee for Clinical Laboratory Standards, Villanova, USA.

15. Bernard L, Vaudaux P, Rohner P, Huggler E, Armanet $\mathrm{M}$, Pittet $\mathrm{D}$, et al. Comparative analysis and validation of different assays for glycopeptide susceptibility among methicillin-resistant Staphylococcus aureus strains. J Microbiol Methods. 2004; 57:231-9.

16. Wootton M, MacGowan AP, Walsh TR, Howe RA. A multicenter study evaluating the current strategies for isolating Staphylococcus aureus strains with reduced susceptibility to glycopeptides. J Clin Microbiol. 2007; 45:329-32.

17. Lulitanond A, Chanawong A, Sribenjalux P, Kaewkes W, Vorachit M, Chongtrakool P, et al. Detection of heterogeneous, intermediate-vancomycin-resistant Staphylococcus aureus (hVISA) using lowconcentration vancomycin disks. Southeast Asian J Trop Med Public Health. 2006; 37:761-7. 